\title{
Possible role of DaVinci Robot in uterine transplantation
}

\author{
Christos Iavazzo ${ }^{1}$, Ioannis D. Gkegkes ${ }^{2}$ \\ 'Department of Gynecological Oncology, Christie Hospital, Manchester, United Kingdom \\ ${ }^{2}$ Department of Surgery, General Hospital of Attica "KAT", Athens, Greece
}

\section{Abstract}

Minimally invasive surgery, specifically robotic surgery, became a common technique used by gynecological surgeons over the last decade. The realization of the first human uterine transplantation commenced new perspectives in the treatment of uterine agenesia or infertility in women with history of hysterectomy at a young age. Robot-assisted technique may enhance the safety of the procedure by facilitating the microvascular anastomosis, vaginal anastomosis, and ligaments' fixation. This study proposes the formation of a multicenter collaboration group to organize a protocol with the aim to clarify the possible role of robotic surgery in uterine transplantation. (J Turk Ger Gynecol Assoc 2015; 16: 179-80)

Keywords: Uterine transplantation, da Vinci® Robot, ethics, robotics

Received: 13 March, 2015

Accepted: 05 May, 2015

Available Online Date: 14 July, 2015

\section{Introduction}

The first human uterine transplantation was attempted in Saudi Arabia in 2002. However, necrosis of the graft occurred 99 days after surgery; thus, the attempt failed (1) that was followed by a lot of criticism. The second case was a 21-year-old patient with Mayer-Rokitansky-Kuster-Hauser syndrome in Turkey who achieved normal cycles and an implanted embryo post transplantation (2). Moreover, recently nine women in Sweden successfully received a transplanted uterus (3). The 6-month results of the first clinical uterus transplantation trial revealed that the durations of donor and recipient surgery ranged from 10 to $13 \mathrm{~h}$ and from 4 to $6 \mathrm{~h}$, respectively. No immediate perioperative complications occurred in any of the recipients, and after 6 months, seven uteri remained viable with regular menses. Mild rejection episodes occurred in four of these patients that were effectively reversed by corticosteroids. The two losses of grafts were because of acute bilateral thrombotic uterine artery occlusions and persistent intrauterine infection (3). The indications of uterine transplantation include women with a history of hysterectomy at a young age for malignant uterine tumors or benign diseases, such as fibroids or adenomyosis; history of emergency peripartum hysterectomy; and history of congenital uterine infertility e.g., Mayer-Rokitansky-Kuster-Hauser syndrome (4).

Robotic surgery became a common technique used by gynecological surgeons over the last decade $(5,6)$. Three-dimensional view, improved dexterity, infiltration of a surgeon's natural tremor, and less operator fatigue are the main advantages of robot-assisted surgery $(7,8)$. In contrast, robotic surgery has some drawbacks, among which increased costs, requirement for a larger operating room because of the bulky machinery, and necessity of a specific training for the surgical team.

In the literature, only 12 cases of uterine transplantation have been described, all of which have been approached with the open technique (1-3, 9). The robot-assisted approach was never described or proposed in the field of uterus transplantation. Therefore, we suggest a new (robotic) approach in the already challenging uterine transplantation. The aim is to form a multicenter collaboration group to organize protocols in animal models as well as humans after ethical approval to clarify the possible role of da Vinci ${ }^{\circledR}$ Robot in uterine transplantation. Two different protocols are proposed: initially, one for animal models, such as sheep or non-human primates and the second one for humans after the possible success in the animal models. Specifically, the sheep and non-human models have been demonstrated to be superior models to that of the pig because the uterus has a relatively smaller size and the vasculature is of similar dimension as that of humans $(10,11)$. The lack of experience in the field and the small number of suitable candidates for the procedure renders the need of a multicenter approach essential.

The surgical technique should include the following steps:

- Hysterectomy of the donor uterus with preserved uterine vessels all the way to internal iliacs plus extended round and uterosacral ligaments.

- Ex vivo preparation of vessels.

- In lithotomy position, the laparoscope arm is introduced through a 12-mm transumbilical trocar with either an open technique or with a direct 4 - puncture technique. In a $25^{\circ}-$ 
$30^{\circ}$ Trandelenburg position, three $8-\mathrm{mm}$ trocars are inserted under direct visualization at the left and right iliac fossae. A $10-\mathrm{mm}$ trocar is positioned 2-cm cranial to the umbilicus and midway to the left trocar. An accessory 5- or 10-mm trocar can be placed midway and a 2 -cm cranial to the umbilicus and right trocar in case of obese patient. In cases of patients with adhesions or large uteri, an extra 5- or 10-mm trocar may be positioned $10 \mathrm{~cm}$ lateral and caudal to the right $8-\mathrm{mm}$ trocar. All trocars are separated by a distance of 8-10 $\mathrm{cm}$ to avoid instrument crowding.

- Entry of the graft through the vaginal wall.

- The vaginal wall of the excised uterus is anastomosed with the vaginal stump using the prosthetic valve-suturing technique used in cardiac valve replacement.

- Vascular anastomosis (internal iliac arteries of the donor uterus that are end-to-end anastomosed with that on the recipient as well as the uterine veins that are end-to-end anastomosed with internal iliac veins and proximal utero-ovarian veins).

- Fixation of ligaments.

The concept of uterus transplantation has already raised various ethical concerns (12). Organ transplantations are considered as life-saving operations or at least as an intervention that offer a significant improvement of a recipient's quality of life (13). Despite the fact that uterus transplantation does not offer health benefit for the recipient, the birth of a child may have an important impact on the quality of life of a woman, entering in this way in the definition and the scope of classic transplant. Moreover, the social acceptance of women with infertility depends on the cultural background of every society. In particular, in Islamic countries, the social respect for women is directly correlated with the childbearing ability of the woman. This particular cultural characteristic was probably the reason why the first uterus transplantation was performed in an Arab country (1). Nevertheless, uterus transplantation can be perceived as "reverse surrogacy," a form of surrogate motherhood where the received uterus takes the role of the surrogate in the recipient's body (13).

The main advantages of such a protocol would be the possible easier technique of micro-vascular anastomosis, three-dimensional (3D) view, wrist-like motion of the robotic arms, possible lower blood loss, possible fewer wound complications, reduced length of hospital stay, and faster return to normal activities (14). However, some could argue against such a technique by highlighting the disadvantages and possible limitations; for example, the decision regarding how the donor uterus is going to be inserted in the abdominal cavity, the fact that there is no previous experience, and the continuous argument of ethical concerns regarding uterine transplantation. Therefore, animal studies are initially suggested. It is necessary for an experienced multidisciplinary transplantation team to extensively evaluate the recipient and donor and to obtain informed consent after discussing the major risks related to surgery, immunosuppression, and possible future pregnancy. Candidates for this operation should be carefully selected. To achieve the optimal possible results, a multispecialty approach is suggested, including careful assessment of the patient by an obstetrician, a gynecologist who is well-trained in both robotic gynecologic surgery and uterine transplantation, a vascular surgeon, an anesthetist, and a psychologist.

\section{Conclusion}

We believe that the use of a robot-assisted technique may enhance the safety of the procedure by facilitating the microvascular anastomosis, vaginal anastomosis, and ligaments fixation. Reduction of the parietal trauma to a minimum, results in a cosmetic and functional outcome that is likely to be superior to that of open laparoscopic techniques. Future studies will be necessary to confirm the long-term safety of such protocols.

Peer-review: Externally peer-reviewed.

Author Contributions: Concept - C.I.; Design - C.I., I.D.G.; Supervision C.I., Resource - C.I., I.D.G.; Materials - C.I., I.D.G.; Data Collection and/or Processing - I.D.G.; Analysis and/or Interpretation - C.I., I.D.G.; Literature Search - I.D.G; Writing - C.I., I.D.G.; Critical Reviews - C.I., I.D.G.

Conflict of Interest: No conflict of interest was declared by the authors.

Financial Disclosure: The authors declared that this study has received no financial support.

\section{References}

1. Fageeh W, Raffa H, Jabbad H, Marzouki A. Transplantation of the human uterus. Int J Gynaecol Obstet 2002; 76: 245-51. [CrossRef]

2. Ozkan O, Akar ME, Ozkan O, Erdogan O, Hadimioglu N, Yilmaz M, et al. Preliminary results of the first human uterus transplantation from a multiorgan donor. Fertil Steril 2013; 99: 470-76. [CrossRef]

3. Brännström M, Johannesson L, Dahm-Kähler P, Enskog A, Mölne J, Kvarnström N, et al. First clinical uterus transplantation trial: a six-month report. Fertil Steril 2014; 101: 1228-36. [CrossRef]

4. Kisu I, Banno K, Mihara M, Hara H, Kato Y, Suganuma N, Aoki Det. Uterine transplantation in primates: a mini-review of the literature. Transplant Proc 2014; 46: 1212-16. [CrossRef]

5. Iavazzo C, Gkegkes ID. Robotic retroperitoneal lymph node dissection in gynaecological neoplasms: comparison of extraperitoneal and transperitoneal lymphadenectomy. Arch Gynecol Obstet 2015 19. [Crossref]

6. Iavazzo C, Gkegkes ID. The role of uterine manipulators in endometrial cancer recurrence after laparoscopic or robotic procedures. Arch Gynecol Obstet 2013; 288: 1003-9. [Crossref]

7. Krill LS, Bristow RE. Robotic surgery: gynecologic oncology. Cancer J 2013; 19: 167-76. [CrossRef]

8. Ohuchida K, Hashizume M. Robotic surgery for cancer. Cancer J 2013; 19: 130-2. [CrossRef]

9. Akar ME, Ozkan O, Aydinuraz B, Dirican K, Cincik M, Mendilcioglu $\mathrm{I}$, et al. Clinical pregnancy after uterus transplantation. Fertil Steril 2013; 100: 1358-63. [CrossRef]

10. Brännström M, Diaz-Garcia C, Hanafy A, Olausson M, Tzakis A. Uterus transplantation: animal research and human possibilities. Fertil Steril 2012; 97: 1269-76. [CrossRef]

11. Akar ME. Might uterus transplantation be an option for uterine factor infertility? J Turk Ger Gynecol Assoc 2015; 16: 45-8. [CrossRef]

12. Milliez J. Uterine transplantation FIGO Committee for the Ethical Aspects of Human Reproduction and Women's Health. Int J Gynaecol Obstet 2009; 106: 270. [CrossRef]

13. Benagiano G, Landeweerd L, Brosens I. Medical and ethical considerations in uterus transplantation. Int J Gynaecol Obstet 2013; 123: 173-7. [CrossRef]

14. Alazzam M, Gillespie A, Hewitt M. Robotic surgery in the management of cervical carcinoma. Arch Gynecol Obstet 2011; 284: 93743. [CrossRef] 